\title{
Torsion units of integral group ring of the simple group $S_{4}(4)$
}

\author{
Antonio L. Rosa
}




\title{
TORSION UNITS OF INTEGRAL GROUP RING OF THE SIMPLE GROUP $S_{4}(4)$
}

\author{
ANTONIO L. ROSA
}

Received 12 June, 2014

\begin{abstract}
We consider the Zassenhaus conjecture for the normalized unit group of the integral group ring of the sympletic simple group $S_{4}(4)$. As a consequence, we confirm for this group the prime graph conjecture.
\end{abstract}

2010 Mathematics Subject Classification: 16S34; 20C05; 20D08

Keywords: Zassenhaus conjecture, torsion unit, partial augmentation, integral group ring

\section{INTRODUCTION, CONJECTURES AND MAIN RESULTS}

In the integral group ring $\mathbb{Z} G$, where $G$ is a finite group, we consider the group of normalized units

$$
V(\mathbb{Z} G)=\left\{u=\sum_{g \in G} \alpha_{g} g \in U(\mathbb{Z} G) \mid \varepsilon(u)=\sum_{g \in G} \alpha_{g}=1\right\} .
$$

A conjecture of Zassenhaus (ZC) states that:

(ZC) Every torsion unit $u \in V(\mathbb{Z} G)$ is conjugate in the rational group algebra $\mathbb{Q} G$ to an element $g \in G$. That is, for every $u \in V(\mathbb{Z} G)$, there exists $v \in \mathbb{Q} G$ such that $v u v^{-1}=g \in G$.

The main tool in the investigation of the Zassenhaus conjecture (ZC) for finite groups with small numbers of normal subgroups is the Luthar-Passi method, which appeared in [24] to show (ZC) in the case where $G=A_{5}$. M. Hertweck (see [19,20]) applied the theory of Brauer characters to the Luthar-Passi method and used it to study the conjecture of Zassenhaus (ZC) for $G=P S L\left(2, p^{n}\right)$.

Now the Luthar-Passi method is very useful to study of $(\mathbf{Z C})$ for arbitrary groups. As recent results, we cite [4,18,21-23, 25-27]. Now, we need to introduce some notation. For $P_{d}(H)$ denote the set of all prime divisors of the orders of elements of torsion part $t(H)$ of a group $H$. The prime graph (or Gruenberg-Kegel graph) of $H$, denoted by $\pi(H)$, is the graph with vertices as the primes in $P_{d}(H)$ and we connect of $p$ to $q$ if there is an element of order $p q$ in the torsion part $t(H)$. In [23] was proposed the following conjecture, 
(PGC) If $\mathrm{G}$ is a finite group then $\pi(G)=\pi(V(\mathbb{Z} G))$.

Clearly, this conjecture is a weaker version of the conjecture of Zassenhaus (ZC). In [23], W. Kimerle showed that (PGC) holds for finite Frobenius groups and solvable groups. V. Bovdi and M. Hertweck in [3] completed the p-version of the Zassenhaus conjecture for Frobenius groups. In [2,5-15] the conjecture (PGC) was studied for several simple sporadic groups.

Here, we are investigating the conjecture (ZC) for the symplectic simple group $S_{4}(4)$. Using the Luthar-Passi method, we can not prove the rational conjugacy (ZC) for all torsion units in $V\left(\mathbb{Z} S_{4}(4)\right)$, but for units of order $\{3,5,17\}$ we are able to prove (ZC). Also our main result gives a lot of information about the partial augmentations of some units. Finally, as a consequence we obtain that (PGC) is valid for this group.

Let $G=S_{4}(4)$. It is well known (see [17]) that $|G|=2^{8} \cdot 3^{2} \cdot 5^{2} \cdot 17$ and $\exp (G)=$ $2^{2} \cdot 3 \cdot 5 \cdot 17$. Let

$$
\begin{aligned}
\mathcal{E}=\{ & C_{1}, C_{2 a}, C_{2 b}, C_{2 c}, C_{3 a}, C_{3 b}, C_{4 a}, C_{4 b}, C_{5 a}, C_{5 b}, C_{5 c}, C_{5 d}, C_{5 e}, C_{6 a}, C_{6 b}, \\
& \left.C_{10 a}, C_{10 b}, C_{10 c}, C_{10 d}, C_{15 a}, C_{15 b}, C_{15 c}, C_{15 d}, C_{17 a}, C_{17 b}, C_{17 c}, C_{17 d}\right\}
\end{aligned}
$$

be the collection of all conjugacy classes of $S_{4}(4)$, where the first index denotes the order of the elements of this conjugacy class and $C_{1}=\{1\}$. Suppose $u=\sum \alpha_{g} g \in$ $V(\mathbb{Z} G)$ has finite order $k$. Denote by

$$
v_{n t}=v_{n t}(u)=\varepsilon_{C_{n t}}(u)=\sum_{g \in C_{n t}} \alpha_{g}
$$

the partial augmentation of $u$ with respect to $C_{n t}$. From the Berman-Higman Theorem (see [1]) one knows that $v_{1}=\alpha_{1}=0$ and

$$
\sum_{C_{n t} \in \mathcal{C}} v_{n t}=1
$$

Hence, for any character $\chi$ of $G$, we get that $\chi(u)=\sum v_{n t} \chi\left(h_{n t}\right)$, where $h_{n t}$ is a representative of the conjugacy class $C_{n t}$.

Our main result is the following

Theorem 1. Let $G$ denote the symplectic group $S_{4}(4)$. Let $u$ be a torsion unit of $V(\mathbb{Z} G)$ of order $|u|$. Denote by $\mathfrak{P}(u)$ the tuple

$$
\begin{gathered}
\left(v_{2 a}, v_{2 b}, v_{2 c}, v_{3 a}, v_{3 b}, v_{4 a}, v_{4 b}, v_{5 a}, v_{5 b}, v_{5 c}, v_{5 d}, v_{5 e}, v_{6 a}, v_{6 b}, v_{10 a},\right. \\
\left.v_{10 b}, v_{10 c}, v_{10 d}, v_{15 a}, v_{15 b}, v_{15 c}, v_{15 d}, v_{17 a}, v_{17 b}, v_{17 c}, v_{17 d}\right) \in \mathbb{Z}^{26}
\end{gathered}
$$

of partial augmentations of $u$ in $V(\mathbb{Z} G)$. The following properties hold.

(i) There is no elements of orders $34,51,85$ in $V(\mathbb{Z} G)$.

(ii) If $|u| \in\{3,5,17\}$, then $u$ is rationally conjugate to some $g \in G$.

(iii) If $|u|=2$, then all components of $\mathfrak{P}(u)$ are zero except possibly $v_{2 a}, v_{2 b}$ and $v_{2 c}$, and the tuple $\left(v_{2 a}, v_{2 b}, v_{2 c}\right)$ is one of

$$
\{(1,-3,3),(2,-2,1),(1,-2,2),(0,-2,3),(3,-1,-1),(2,-1,0) \text {, }
$$




$$
\begin{gathered}
(1,-1,1),(0,-1,2),(-1,-1,3),(2,0,-1),(1,0,0),(0,0,1), \\
(-1,0,2),(-2,0,3),(1,1,-1),(0,1,0),(-1,1,1),(-2,1,2), \\
(-3,1,3),(0,2,-1),(-1,2,0),(-2,2,1),(-1,3,-1)\} .
\end{gathered}
$$

As an immediate consequence of part (i) of the Theorem we obtain

Corollary 1. If $G=S_{4}(4)$ then $\pi(G)=\pi(V(\mathbb{Z} G))$.

\section{Preliminaries}

The following result is a reformulation of the Zassenhaus conjecture in terms of vanishing of partial augmentations of torsion units.

Proposition 1 (see [24]). Let $u \in V(\mathbb{Z} G)$ be of order $k$. Then $u$ is conjugate in $\mathbb{Q} G$ to an element $g \in G$ if and only if for each d dividing $k$ there is precisely one conjugacy class $C$ with partial augmentation $\varepsilon_{C}\left(u^{d}\right) \neq 0$.

This fact shows that several partial augmentations are zero.

Proposition 2 (see [19], Proposition 3.1; [20], Proposition 2.2). Let $G$ be a finite group and let $u$ be a torsion unit in $V(\mathbb{Z} G)$. If $x$ is an element of $G$ whose p-part, for some prime $p$, has order strictly greater than the order of the $p$-part of $u$, then $\varepsilon_{x}(u)=0$.

The key restriction on partial augmentations is given by the following result that is the cornerstone of the Luthar-Passi method.

Proposition 3 (see $[20,24])$. Let either $p=0$ or $p$ a prime divisor of $|G|$. Suppose that $u \in V(\mathbb{Z} G)$ has finite order $k$ and assume $k$ and $p$ are coprime in case $p \neq 0$. If $z$ is a complex primitive $k$-th root of unity and $\chi$ is either a classical character or a $p$-Brauer character of $G$, then for every integer $l$ the number

$$
\mu_{l}(u, \chi, p)=\frac{1}{k} \sum_{d \mid k} \operatorname{Tr}_{\mathbb{Q}\left(z^{d}\right) / \mathbb{Q}}\left\{\chi\left(u^{d}\right) z^{-d l}\right\}
$$

is a non-negative integer.

Note that if $p=0$, we will use the notation $\mu_{l}(u, \chi, *)$ for $\mu_{l}(u, \chi, 0)$.

Finally, we shall use the well-known bound for orders of torsion units.

Proposition 4 (see [16]). The order of a torsion element $u \in V(\mathbb{Z} G)$ is a divisor of the exponent of $G$.

\section{Proof of the Theorem}

Throughout this section we denote $S_{4}(4)$ by $G$. The character table of $G$, as well as the $p$-Brauer character tables, which will be denoted by $\mathfrak{B C T}(p)$ where $p \in$ $\{2,3,5,17\}$, can be found using the computational algebra system GAP [17]. For the 
characters and conjugacy classes we will use throughout the paper the same notation, indexation inclusive, as used in the GAP Character Table Library.

First of all we start to investigate units of orders 2, 3, 5 and 17, since the group $G$ possesses elements of these orders. After this, by Proposition 4, the order of each torsion unit divides the exponent of $G$, so to prove the Kimmerle's conjecture, it remains to consider units of orders 34,51 and 85 . We prove that no units of all these orders do appear in $V(\mathbb{Z} G)$.

Now we consider each case of possible orders separately.

Let $u$ be an involution. By (1.1) and Proposition 2 we get

$$
v_{2 a}+v_{2 b}+v_{2 c}=1 \text {. }
$$

Put $t_{1}=3 v_{2 a}+3 v_{2 b}-v_{2 c}$ and $t_{2}=5 v_{2 a}-3 v_{2 b}+v_{2 c}$. By (2.1) we obtain the system of inequalities

$$
\begin{array}{ll}
\mu_{1}\left(u, \chi_{2}, *\right)=\frac{1}{2}\left(2 t_{1}+18\right) \geq 0 ; & \mu_{0}\left(u, \chi_{2}, *\right)=\frac{1}{2}\left(-2 t_{1}+18\right) \geq 0 ; \\
\mu_{0}\left(u, \chi_{3}, *\right)=\frac{1}{2}\left(2 t_{2}+34\right) \geq 0 ; & \mu_{1}\left(u, \chi_{3}, *\right)=\frac{1}{2}\left(-2 t_{2}+34\right) \geq 0,
\end{array}
$$

from which $-9 \leq t_{1} \leq 9$ and $-17 \leq t_{2} \leq 17$. Furthermore, from the system of linear equations

$$
v_{2 a}+v_{2 b}+v_{2 c}=1, \quad 3 v_{2 a}+3 v_{2 b}-v_{2 c}=t_{1}, \quad 5 v_{2 a}-3 v_{2 b}+v_{2 c}=t_{2},
$$

we select only integer solutions, and using the condition that all $\mu_{i}\left(u, \chi_{j}, *\right)$ are nonnegative integers, we obtain twenty three tuples $\left(v_{2 a}, v_{2 b}, v_{2 c}\right)$.

Let $u$ be a unit of order 3. By (1.1) and Proposition 2 we get

$$
v_{3 a}+v_{3 b}=1 \text {. }
$$

Put $t_{1}=v_{3 a}+4 v_{3 b}$. Using $\mathfrak{B C T}(2)$ and by (2.1) we obtain the system of inequalities

$$
\mu_{0}\left(u, \chi_{7}, 2\right)=\frac{1}{3}\left(2 t_{1}+16\right) \geq 0, \quad \mu_{1}\left(u, \chi_{7}, 2\right)=\frac{1}{3}\left(-t_{1}+16\right) \geq 0,
$$

from which $t_{1} \in\{1+3 k \mid-3 \leq k \leq 5\}$. Using inequalities

$\mu_{0}\left(u, \chi_{2}, 2\right)=\frac{1}{3}\left(-4 v_{3 a}+2 v_{3 b}+4\right) \geq 0, \quad \mu_{0}\left(u, \chi_{4}, 2\right)=\frac{1}{3}\left(2 v_{3 a}-4 v_{3 b}+4\right) \geq 0$,

we obtain only two integral solutions $\left(v_{3 a}, v_{3 b}\right) \in\{(0,1),(1,0)\}$.

Let $u$ be a unit of order 5. By (1.1) and Proposition 2 we get

$$
v_{5 a}+v_{5 b}+v_{5 c}+v_{5 d}+v_{5 e}=1 \text {. }
$$

Put

$$
\alpha=3 v_{5 a}+3 v_{5 b}+3 v_{5 c}+3 v_{5 d}-2 v_{5 e} \text { and } \beta=4 v_{5 a}+4 v_{5 b}-v_{5 c}-v_{5 d}-v_{5 e} .
$$

Using $\mathfrak{B C T}(2)$ and by (2.1) we obtain the system of inequalities

$$
\begin{array}{ll}
\mu_{0}\left(u, \chi_{2}, *\right)=\frac{1}{5}(4 \alpha+18) \geq 0 ; & \mu_{1}\left(u, \chi_{2}, *\right)=\frac{1}{5}(-\alpha+18) \geq 0 ; \\
\mu_{0}\left(u, \chi_{3}, *\right)=\frac{1}{5}(4 \beta+34) \geq 0 ; & \mu_{0}\left(u, \chi_{6}, 2\right)=\frac{1}{5}(-4 \beta+16) \geq 0,
\end{array}
$$


so $\alpha \in\{-2,3,8,13,18\}$ and $\beta \in\{-6,-1,4\}$ and we obtain only five integral solutions

$\left(v_{5 a}, v_{5 b}\right) \in\{(1,0,0,0,0),(0,0,0,0,1),(0,0,0,1,0),(0,0,1,0,0),(0,1,0,0,0)\}$.

Let $u$ be a unit of order 17. By (1.1) and Proposition 2 we get

$$
v_{17 a}+v_{17 b}+v_{17 c}+v_{17 d}=1 .
$$

Put

$$
\begin{gathered}
t_{1}=13 v_{17 a}-4 v_{17 b}-4 v_{17 c}-4 v_{17 d}, \quad t_{2}=4 v_{17 a}-13 v_{17 b}+4 v_{17 c}+4 v_{17 d}, \\
\text { and } t_{3}=4 v_{17 a}+4 v_{17 b}-13 v_{17 c}+4 v_{17 d} .
\end{gathered}
$$

By (2.1) we have

$$
\begin{array}{rlrl}
\mu_{1}\left(u, \chi_{2}, 2\right) & =\frac{1}{17}\left(t_{1}+4\right) \geq 0 ; & \mu_{3}\left(u, \chi_{12}, 2\right) & =\frac{1}{17}\left(-t_{1}+64\right) \geq 0 ; \\
\mu_{6}\left(u, \chi_{12}, 2\right) & =\frac{1}{17}\left(t_{2}+64\right) \geq 0 ; & & \mu_{2}\left(u, \chi_{2}, 2\right)=\frac{1}{17}\left(-t_{2}+4\right) \geq 0 ; \\
\mu_{2}\left(u, \chi_{12}, 2\right)=\frac{1}{17}\left(t_{3}+64\right) \geq 0 ; & \mu_{3}\left(u, \chi_{2}, 2\right)=\frac{1}{17}\left(-t_{3}+4\right) \geq 0 .
\end{array}
$$

This yields

$$
\begin{gathered}
t_{1} \in\{-4,13,30,47,64\}, \quad t_{2} \in\{-64,-47,-30,-13,4\}, \\
\text { and } t_{3} \in\{-64,-47,-30,-13,4\} .
\end{gathered}
$$

Using inequality

$$
\mu_{6}\left(u, \chi_{2}, 2\right)=\frac{1}{17}\left(-4 v_{17 a}-4 v_{17 b}-4 v_{17 c}+13 v_{17 d}+4\right) \geq 0
$$

we have the four trivial solutions.

Let $u$ be a unit of order 34. By (1.1) and Proposition 2 we have

$$
v_{2 a}+v_{2 b}+v_{2 c}+v_{17 a}+v_{17 b}+v_{17 c}+v_{17 d}=1 \text {. }
$$

Put

$$
\begin{gathered}
t_{1}=6 v_{2 a}+6 v_{2 b}-2 v_{2 c}-v_{17 a}-v_{17 b}-v_{17 c}-v_{17 d}, \\
t_{2}=5 v_{2 a}-3 v_{2 b}+v_{2 c}, \quad t_{3}=3 v_{2 a}-5 v_{2 b}-v_{2 c}, \\
t_{4}=15 v_{2 a}+15 v_{2 b}-v_{2 c}-4 v_{17 a}+13 v_{17 b}-4 v_{17 c}-4 v_{17 d} \\
t_{5}=15 v_{2 a}+15 v_{2 b}-v_{2 c}+13 v_{17 a}-4 v_{17 b}-4 v_{17 c}-4 v_{17 d} \\
t_{6}=15 v_{2 a}+15 v_{2 b}-v_{2 c}-4 v_{17 a}-4 v_{17 b}-4 v_{17 c}+13 v_{17 d}
\end{gathered}
$$

Since $\left|u^{17}\right|=2$, for any character $\chi$ of $G$ we need to consider twenty three cases, defined by part (ii) of the Theorem:

(1) Let

$$
\begin{array}{r}
\chi\left(u^{17}\right) \in\{\chi(2 a), \chi(2 a)-2 \chi(2 b)+2 \chi(2 c), 2 \chi(2 a)-\chi(2 b),-\chi(2 b)+2 \chi(2 c), \\
\chi(2 b),-\chi(2 a)+2 \chi(2 c),-2 \chi(2 a)+\chi(2 b)+2 \chi(2 c),-\chi(2 a)+2 \chi(2 b)\} .
\end{array}
$$

Then by (2.1) we obtain two incompatible inequalities

$$
\mu_{17}\left(u, \chi_{2}, *\right)=\frac{1}{34}\left(16 t_{1}+\alpha\right) \geq 0, \quad \mu_{0}\left(u, \chi_{2}, *\right)=\frac{1}{34}\left(-16 t_{1}+\beta\right) \geq 0,
$$


where $(\alpha, \beta) \in\{(40,28),(24,44)\}$.

(2) Let

$$
\begin{array}{r}
\chi\left(u^{17}\right) \in\{\chi(2 c), 2 \chi(2 a)-2 \chi(2 b)+\chi(2 c), \chi(2 a)-\chi(2 b)+\chi(2 c), \\
-\chi(2 a)+\chi(2 b)+\chi(2 c),-2 \chi(2 a)+2 \chi(2 b)+\chi(2 c)\} .
\end{array}
$$

Using (2.1), we obtain the incompatible system of inequalities

$$
\begin{aligned}
\mu_{17}\left(u, \chi_{2}, *\right) & =\frac{1}{34}\left(16 t_{1}+32\right) \geq 0 ; \\
\mu_{0}\left(u, \chi_{2}, *\right) & =\frac{1}{34}\left(-16 t_{1}+36\right) \geq 0 ; \\
\mu_{1}\left(u, \chi_{2}, *\right) & =\frac{1}{34}\left(-t_{1}+15\right) \geq 0 .
\end{aligned}
$$

(3) Let

$$
\begin{array}{r}
\chi\left(u^{17}\right) \in\{-2 \chi(2 b)+3 \chi(2 c),-2 \chi(2 a)+3 \chi(2 c), \\
2 \chi(2 a)-\chi(2 c), 2 \chi(2 b)-\chi(2 c)\} .
\end{array}
$$

Using (2.1), we obtain the system of inequalities

$\mu_{0}\left(u, \chi_{3}, *\right)=\frac{1}{34}\left(32 t_{2}+\alpha\right) \geq 0 ; \quad \mu_{17}\left(u, \chi_{3}, *\right)=\frac{1}{34}\left(-32 t_{2}+\beta\right) \geq 0$,

where $(\alpha, \beta) \in\{(52,16),(20,48)\}$, which has no integral solution.

(4) Let $\chi\left(u^{17}\right)=\chi(2 a)-3 \chi(2 b)+3 \chi(2 c)$. Using (2.1), we calculate the following system of inequalities

$$
\begin{array}{rlrl}
\mu_{17}\left(u, \chi_{2}, *\right) & =\frac{1}{34}\left(16 t_{1}+16\right) \geq 0 ; & \mu_{1}\left(u, \chi_{2}, *\right) & =\frac{1}{34}\left(-t_{1}-1\right) \geq 0 ; \\
\mu_{1}\left(u, \chi_{3}, *\right) & =\frac{1}{34}\left(2 t_{2}\right) \geq 0 ; & & \mu_{17}\left(u, \chi_{3}, *\right)=\frac{1}{34}\left(-32 t_{2}\right) \geq 0 ; \\
\mu_{2}\left(u, \chi_{4}, *\right)=\frac{1}{34}\left(2 t_{3}+4\right) \geq 0 ; & \mu_{0}\left(u, \chi_{4}, *\right) & =\frac{1}{34}\left(-32 t_{3}+4\right) \geq 0 ; \\
\mu_{4}\left(u, \chi_{17}, *\right) & =\frac{1}{34}\left(t_{4}+271\right) \geq 0 ; & & \mu_{1}\left(u, \chi_{17}, *\right)=\frac{1}{34}\left(-t_{4}+205\right) \geq 0 ; \\
\mu_{2}\left(u, \chi_{17}, *\right) & =\frac{1}{34}\left(t_{5}+254\right) \geq 0 ; & & \mu_{9}\left(u, \chi_{17}, *\right)=\frac{1}{34}\left(-t_{5}+188\right) \geq 0 ; \\
\mu_{12}\left(u, \chi_{17}, *\right) & =\frac{1}{34}\left(t_{6}+254\right) \geq 0 ; & & \mu_{3}\left(u, \chi_{17}, *\right)=\frac{1}{34}\left(-t_{6}+188\right) \geq 0 .
\end{array}
$$

It follows that $t_{1}=-1, t_{2}=0, t_{3}=-2$,

$$
t_{4} \in\{1+34 k \mid-8 \leq k \leq 6\}, t_{5}, t_{6} \in\{18+34 k \mid-8 \leq k \leq 5\},
$$

and we have no solutions.

(5) Let $\chi\left(u^{17}\right)=3 \chi(2 a)-\chi(2 b)-\chi(2 c)$. Using (2.1), we obtain the system of inequalities

$$
\begin{aligned}
\mu_{2}\left(u, \chi_{2}, *\right) & =\frac{1}{34}\left(t_{1}+3\right) \geq 0 ; & \mu_{0}\left(u, \chi_{2}, *\right) & =\frac{1}{34}\left(-16 t_{1}+20\right) \geq 0 \\
\mu_{1}\left(u, \chi_{3}, *\right) & =\frac{1}{34}\left(2 t_{2}\right) \geq 0 ; & \mu_{17}\left(u, \chi_{3}, *\right) & =\frac{1}{34}\left(-32 t_{2}\right) \geq 0 ; \\
\mu_{2}\left(u, \chi_{4}, *\right) & =\frac{1}{34}\left(2 t_{3}+4\right) \geq 0 ; & \mu_{0}\left(u, \chi_{4}, *\right) & =\frac{1}{34}\left(-32 t_{3}+4\right) \geq 0 ; \\
\mu_{4}\left(u, \chi_{17}, *\right) & =\frac{1}{34}\left(t_{4}+190\right) \geq 0 ; & \mu_{1}\left(u, \chi_{17}, *\right) & =\frac{1}{34}\left(-t_{4}+252\right) \geq 0 \\
\mu_{2}\left(u, \chi_{17}, *\right) & =\frac{1}{34}\left(t_{5}+190\right) \geq 0 ; & \mu_{9}\left(u, \chi_{17}, *\right) & =\frac{1}{34}\left(-t_{5}+252\right) \geq 0
\end{aligned}
$$


$\mu_{12}\left(u, \chi_{17}, *\right)=\frac{1}{34}\left(t_{6}+207\right) \geq 0 ; \quad \mu_{3}\left(u, \chi_{17}, *\right)=\frac{1}{34}\left(-t_{6}+269\right) \geq 0$.

It follows that $t_{1}=-3, t_{2}=0, t_{3}=-2$,

$t_{4}, t_{5} \in\{14+34 k \mid-6 \leq k \leq 7\}, t_{6} \in\{31+34 k \mid-7 \leq k \leq 7\}$,

and we have no solutions again.

(6) Let $\chi\left(u^{17}\right)=-\chi(2 a)-\chi(2 b)+3 \chi(2 c)$. Again, using (2.1), we obtain the system of inequalities

$$
\begin{array}{rlrl}
\mu_{17}\left(u, \chi_{2}, *\right) & =\frac{1}{34}\left(16 t_{1}+16\right) \geq 0 ; & \mu_{1}\left(u, \chi_{2}, *\right) & =\frac{1}{34}\left(-t_{1}-1\right) \geq 0 ; \\
\mu_{0}\left(u, \chi_{3}, *\right) & =\frac{1}{34}\left(32 t_{2}+36\right) \geq 0 ; & \mu_{17}\left(u, \chi_{3}, *\right)=\frac{1}{34}\left(-32 t_{2}+32\right) \geq 0 ; \\
\mu_{17}\left(u, \chi_{4}, *\right)=\frac{1}{34}\left(32 t_{3}+32\right) \geq 0 ; & \mu_{0}\left(u, \chi_{4}, *\right)=\frac{1}{34}\left(-32 t_{3}+36\right) \geq 0 ; \\
\mu_{4}\left(u, \chi_{17}, *\right)=\frac{1}{34}\left(t_{4}+254\right) \geq 0 ; & \mu_{1}\left(u, \chi_{17}, *\right)=\frac{1}{34}\left(-t_{4}+188\right) \geq 0 ; \\
\mu_{2}\left(u, \chi_{17}, *\right)=\frac{1}{34}\left(t_{5}+254\right) \geq 0 ; & \mu_{9}\left(u, \chi_{17}, *\right)=\frac{1}{34}\left(-t_{5}+188\right) \geq 0 ; \\
\mu_{12}\left(u, \chi_{17}, *\right)=\frac{1}{34}\left(t_{6}+271\right) \geq 0 ; & \mu_{3}\left(u, \chi_{17}, *\right)=\frac{1}{34}\left(-t_{6}+205\right) \geq 0 .
\end{array}
$$

It follows that $t_{1}=-1, t_{2}=1, t_{3}=-1$,

$$
t_{4}, t_{5} \in\{18+34 k \mid-8 \leq k \leq 5\}, t_{6} \in\{1+34 k \mid-8 \leq k \leq 6\},
$$

and we have no solutions again.

(7) Let $\chi\left(u^{17}\right)=\chi(2 a)+\chi(2 b)-\chi(2 c)$. Then, by (2.1), we obtain the system of inequalities

$$
\begin{array}{rlrl}
\mu_{2}\left(u, \chi_{2}, *\right) & =\frac{1}{34}\left(t_{1}+3\right) \geq 0 ; & & \mu_{0}\left(u, \chi_{2}, *\right)=\frac{1}{34}\left(-16 t_{1}+20\right) \geq 0 ; \\
\mu_{0}\left(u, \chi_{3}, *\right)=\frac{1}{34}\left(32 t_{2}+36\right) \geq 0 ; & \mu_{17}\left(u, \chi_{3}, *\right)=\frac{1}{34}\left(-32 t_{2}+32\right) \geq 0 ; \\
\mu_{17}\left(u, \chi_{4}, *\right)=\frac{1}{34}\left(32 t_{3}+32\right) \geq 0 ; & \mu_{0}\left(u, \chi_{4}, *\right)=\frac{1}{34}\left(-32 t_{3}+36\right) \geq 0 ; \\
\mu_{4}\left(u, \chi_{17}, *\right)=\frac{1}{34}\left(t_{4}+190\right) \geq 0 ; & \mu_{1}\left(u, \chi_{17}, *\right)=\frac{1}{34}\left(-t_{4}+252\right) \geq 0 ; \\
\mu_{2}\left(u, \chi_{17}, *\right)=\frac{1}{34}\left(t_{5}+190\right) \geq 0 ; & \mu_{9}\left(u, \chi_{17}, *\right)=\frac{1}{34}\left(-t_{5}+252\right) \geq 0 ; \\
\mu_{12}\left(u, \chi_{17}, *\right)=\frac{1}{34}\left(t_{6}+207\right) \geq 0 ; & \mu_{3}\left(u, \chi_{17}, *\right)=\frac{1}{34}\left(-t_{6}+269\right) \geq 0 .
\end{array}
$$

It follows that $t_{1}=-3, t_{2}=1, t_{3}=-1$,

$$
t_{4}, t_{5} \in\{14+34 k \mid-6 \leq k \leq 7\}, t_{6} \in\{31+34 k \mid-7 \leq k \leq 7\} \text {, }
$$

and we have no solutions.

(8) Let $\chi\left(u^{17}\right)=-3 \chi(2 a)+\chi(2 b)+3 \chi(2 c)$. Then, by (2.1), we obtain the system of inequalities

$$
\begin{array}{rlrl}
\mu_{17}\left(u, \chi_{2}, *\right) & =\frac{1}{34}\left(16 t_{1}+16\right) \geq 0 ; & & \mu_{1}\left(u, \chi_{2}, *\right)=\frac{1}{34}\left(-t_{1}-1\right) \geq 0 ; \\
\mu_{0}\left(u, \chi_{3}, *\right)=\frac{1}{34}\left(32 t_{2}+4\right) \geq 0 ; & & \mu_{2}\left(u, \chi_{3}, *\right)=\frac{1}{34}\left(-2 t_{2}+4\right) \geq 0 ; \\
\mu_{17}\left(u, \chi_{4}, *\right)=\frac{1}{34}\left(32 t_{3}\right) \geq 0 ; & \mu_{1}\left(u, \chi_{4}, *\right)=\frac{1}{34}\left(-2 t_{3}\right) \geq 0 ; \\
\mu_{4}\left(u, \chi_{17}, *\right)=\frac{1}{34}\left(t_{4}+254\right) \geq 0 ; & \mu_{1}\left(u, \chi_{17}, *\right)=\frac{1}{34}\left(-t_{4}+188\right) \geq 0 ;
\end{array}
$$




$$
\begin{array}{cl}
\mu_{2}\left(u, \chi_{17}, *\right)=\frac{1}{34}\left(t_{5}+271\right) \geq 0 ; & \mu_{9}\left(u, \chi_{17}, *\right)=\frac{1}{34}\left(-t_{5}+205\right) \geq 0 ; \\
\mu_{12}\left(u, \chi_{17}, *\right)=\frac{1}{34}\left(t_{6}+254\right) \geq 0 ; \quad & \mu_{3}\left(u, \chi_{17}, *\right)=\frac{1}{34}\left(-t_{6}+188\right) \geq 0 . \\
\text { It follows that } t_{1}=-1, t_{2}=2, t_{3}=0, & \\
t_{4}, t_{6} \in\{18+34 k \mid-8 \leq k \leq 5\}, t_{5} \in\{1+34 k \mid-8 \leq k \leq 6\}, &
\end{array}
$$

and we have no solutions.

(9) Let $\chi\left(u^{17}\right)=-\chi(2 a)+3 \chi(2 b)-\chi(2 c)$. Then, by (2.1), we obtain the system of inequalities

$$
\begin{array}{rlrl}
\mu_{2}\left(u, \chi_{2}, *\right) & =\frac{1}{34}\left(t_{1}+3\right) \geq 0 ; & & \mu_{0}\left(u, \chi_{2}, *\right)=\frac{1}{34}\left(-16 t_{1}+20\right) \geq 0 ; \\
\mu_{0}\left(u, \chi_{3}, *\right)=\frac{1}{34}\left(32 t_{2}+4\right) \geq 0 ; & & \mu_{2}\left(u, \chi_{3}, *\right)=\frac{1}{34}\left(-2 t_{2}+4\right) \geq 0 ; \\
\mu_{17}\left(u, \chi_{4}, *\right)=\frac{1}{34}\left(32 t_{3}\right) \geq 0 ; & & \mu_{1}\left(u, \chi_{4}, *\right)=\frac{1}{34}\left(-2 t_{3}\right) \geq 0 ; \\
\mu_{4}\left(u, \chi_{17}, *\right)=\frac{1}{34}\left(t_{4}+190\right) \geq 0 ; & & \mu_{1}\left(u, \chi_{17}, *\right)=\frac{1}{34}\left(-t_{4}+252\right) \geq 0 ; \\
\mu_{2}\left(u, \chi_{17}, *\right)=\frac{1}{34}\left(t_{5}+207\right) \geq 0 ; & & \mu_{9}\left(u, \chi_{17}, *\right)=\frac{1}{34}\left(-t_{5}+269\right) \geq 0 ; \\
\mu_{12}\left(u, \chi_{17}, *\right)=\frac{1}{34}\left(t_{6}+190\right) \geq 0 ; & \mu_{3}\left(u, \chi_{17}, *\right)=\frac{1}{34}\left(-t_{6}+252\right) \geq 0 .
\end{array}
$$

It follows that $t_{1}=-3, t_{2}=2, t_{3}=0$,

$$
t_{4}, t_{6} \in\{14+34 k \mid-6 \leq k \leq 7\}, t_{5} \in\{31+34 k \mid-7 \leq k \leq 7\},
$$

and we have no solutions.

Let $u$ be a unit of order 51. By (1.1) and Proposition 2 we have

$$
v_{3 a}+v_{3 b}+v_{17 a}+v_{17 b}+v_{17 c}+v_{17 d}=1 .
$$

Put

$$
t_{1}=v_{17 a}+v_{17 b}+v_{17 c}+v_{17 d} .
$$

Then using (2.1) we obtain the non-compatible system of inequalities

$$
\mu_{0}\left(u, \chi_{2}, *\right)=\frac{1}{51}\left(32 t_{1}+34\right) \geq 0, \quad \mu_{17}\left(u, \chi_{2}, *\right)=\frac{1}{51}\left(-16 t_{1}+34\right) \geq 0 .
$$

Let $u$ be a unit of order 85. By (1.1) and Proposition 2 we have

$$
v_{5 a}+v_{5 b}+v_{5 c}+v_{5 d}+v_{5 e}+v_{17 a}+v_{17 b}+v_{17 c}+v_{17 d}=1 .
$$

Put

$$
t_{1}=3 v_{5 a}+3 v_{5 b}+3 v_{5 c}+3 v_{5 d}-2 v_{5 e}+v_{17 a}+v_{17 b}+v_{17 c}+v_{17 d} .
$$

Since $\left|u^{17}\right|=5$, for any character $\chi$ of $G$ we need to consider five cases, defined by part (iii) of the Theorem:

(1) Let $\chi\left(u^{17}\right) \in\{\chi(5 a), \chi(5 b), \chi(5 c), \chi(5 d)\}$. Using (2.1), we obtain the system of inequalities

$$
\mu_{0}\left(u, \chi_{2}, *\right)=\frac{1}{85}\left(64 t_{1}+46\right) \geq 0, \quad \mu_{17}\left(u, \chi_{2}, *\right)=\frac{1}{85}\left(-16 t_{1}+31\right) \geq 0,
$$

which has no integral solution. 
(2) Let $\chi\left(u^{17}\right)=\chi(5 e)$. Again, using (2.1), we obtain the system of inequalities

$$
\mu_{0}\left(u, \chi_{2}, *\right)=\frac{1}{85}\left(64 t_{1}+26\right) \geq 0, \quad \mu_{5}\left(u, \chi_{2}, *\right)=\frac{1}{85}\left(-4 t_{1}+9\right) \geq 0,
$$

which has no integral solution.

\section{REFERENCES}

[1] V. A. Artamonov and A. A. Bovdi, "Integral group rings: groups of invertible elements and classical K-theory," in Algebra. Topology. Geometry, Vol. 27 (Russian), ser. Itogi Nauki i Tekhniki. Moscow: Akad. Nauk SSSR Vsesoyuz. Inst. Nauchn. i Tekhn. Inform., 1989, pp. 3-43, 232.

[2] V. Bovdi, A. Grishkov, and A. Konovalov, "Kimmerle conjecture for the Held and O'Nan sporadic simple groups," Sci. Math. Jpn., vol. 69, no. 3, pp. 353-361, 2009.

[3] V. Bovdi and M. Hertweck, "Zassenhaus conjecture for central extensions of $S_{5}$," J. Group Theory, vol. 11, no. 1, pp. 63-74, 2008.

[4] V. Bovdi, C. Höfert, and W. Kimmerle, "On the first Zassenhaus conjecture for integral group rings," Publ. Math. Debrecen, vol. 65, no. 3-4, pp. 291-303, 2004.

[5] V. Bovdi and A. Konovalov, "Integral group ring of the first Mathieu simple group," in Groups St. Andrews 2005. Vol. 1, ser. London Math. Soc. Lecture Note Ser. Cambridge Univ. Press, Cambridge, 2007, vol. 339, pp. 237-245.

[6] V. Bovdi and A. Konovalov, "Integral group ring of the Mathieu simple group $M_{24}$," J. Algebra Appl., vol. 11, no. 1, pp. 1250 016, 10, 2012.

[7] V. A. Bovdi, E. Jespers, and A. B. Konovalov, "Torsion units in integral group rings of Janko simple groups," Math. Comp., vol. 80, no. 273, pp. 593-615, 2011.

[8] V. A. Bovdi and A. B. Konovalov, "Integral group ring of the McLaughlin simple group," Algebra Discrete Math., vol. 2, pp. 43-53, 2007.

[9] V. A. Bovdi and A. B. Konovalov, "Integral group ring of the Mathieu simple group $M_{23}$," Comm. Algebra, vol. 36, no. 7, pp. 2670-2680, 2008.

[10] V. A. Bovdi and A. B. Konovalov, "Integral group ring of Rudvalis simple group," Ukraïn. Mat. Zh., vol. 61, no. 1, pp. 3-13, 2009.

[11] V. A. Bovdi and A. B. Konovalov, "Torsion units in integral group ring of Higman-Sims simple group," Studia Sci. Math. Hungar, vol. 47, no. 1, pp. 1-11, 2010.

[12] V. A. Bovdi, A. B. Konovalov, and S. Linton, "Torsion units in integral group ring of the Mathieu simple group $\mathrm{M}_{22}$, , LMS J. Comput. Math., vol. 11, pp. 28-39, 2008.

[13] V. A. Bovdi, A. B. Konovalov, and S. Linton, "Torsion units in integral group rings of Conway simple groups," Internat. J. Algebra Comput., vol. 21, no. 4, pp. 615-634, 2011.

[14] V. A. Bovdi, A. B. Konovalov, and S. Siciliano, "Integral group ring of the Mathieu simple group M12," Rend. Circ. Mat. Palermo (2), vol. 56, no. 1, pp. 125-136, 2007.

[15] V. A. Bovdi, A. B. Konovalov, and E. d. N. Marcos, "Integral group ring of the Suzuki sporadic simple group," Publ. Math. Debrecen, vol. 72, no. 3-4, pp. 487-503, 2008.

[16] J. Cohn and D. Livingstone, "On the structure of group algebras. I," Canad. J. Math., vol. 17, pp. 583-593, 1965.

[17] GAP - Groups, Algorithms, and Programming, Version 4.4.9, The GAP Group, 2006, (http://www.gap-system.org)

[18] J. Gildea, "Zassenhaus conjecture for integral group ring of simple linear groups," J. Algebra Appl., vol. 12, no. 6, pp. 1350 016, 10, 2013.

[19] M. Hertweck, "On the torsion units of some integral group rings," Algebra Colloq., vol. 13, no. 2, pp. 329-348, 2006.

[20] M. Hertweck, "Partial augmentations and Brauer character values of torsion units in group rings," E-print arXiv:math.RA/0612429v2, 2007. 
[21] M. Hertweck, "Torsion units in integral group rings of certain metabelian groups," Proc. Edinb. Math. Soc., vol. 51, no. 2, pp. 363-385, 2008.

[22] C. Höfert and W. Kimmerle, "On torsion units of integral group rings of groups of small order," in Groups, rings and group rings, ser. Lect. Notes Pure Appl. Math. Chapman \& Hall/CRC, Boca Raton, FL, 2006, vol. 248, pp. 243-252.

[23] W. Kimmerle, "On the prime graph of the unit group of integral group rings of finite groups," in Groups, rings and algebras, ser. Contemporary Mathematics. AMS, 2006, vol. 420, pp. 215-228.

[24] I. S. Luthar and I. B. S. Passi, "Zassenhaus conjecture for $A_{5}$," Proc. Indian Acad. Sci. Math. Sci., vol. 99 , no. 1 , pp. $1-5,1989$.

[25] M. A. Salim, "Torsion units in the integral group ring of the alternating group of degree 6," Comm. Algebra, vol. 35, no. 12, pp. 4198-4204, 2007.

[26] M. A. Salim, "Kimmerle's conjecture for integral group rings of some alternating groups," Acta Math. Acad. Paedagog. Nyházi. (N.S.), vol. 27, no. 1, pp. 9-22, 2011.

[27] M. A. Salim, "The prime graph conjecture for integral group rings of some alternating groups," Int. J. Group Theory, vol. 2, no. 1, pp. 175-185, 2013.

\section{Author's address}

\section{Antonio L. Rosa}

Departamento de Matemática, Centro de Ciências Exatas, Universidade Federal do Espírito Santo, Av. Fernando Ferrari, 514, Vitória-ES, 29075-910, Brazil.

E-mail address: antonio.l.rosa@ufes.br, dr_alrosa@hotmail.com 